\title{
Birefringence measurements of sapphire test masses for laser interferometer gravitational wave detector
}

\author{
F. Benabid, M. Notcutt, L. Ju, D.G. Blair \\ Physics Department, University of Western Australia. \\ Nedlands, W.A. 6907 Australia
}

\begin{abstract}
We report measurements of the birefringence of a high purity polished sapphire sample to determine the fringe contrast attainable in a laser interferometer gravitational detector. The results show that birefringence-induced losses in the interferometer would be comparable whether sapphire or silica is used.
\end{abstract}

Key words:

Birefringence, Sapphire, Gravitational wave detector, Beamsplitter,

Photo-elastic modulator.

E-mail: $\quad$ fetah@earwax.pd.uwa.edu.au

Telephone: $\quad+(61)(08) 9380-2749$

Fax: $\quad+(61)(08) 9380-1170$ 


\section{Introduction}

The excellent mechanical properties ${ }^{1,2}$ and low optical absorption of sapphire ${ }^{3}$ make it a possible material for the test masses and beamsplitter in laser interferometric gravitational wave detectors. Advanced interferometers are proposed which will use at least one recycling mirror and a Michelson configuration ${ }^{4}$ where the beamsplitter is within the optical cavities carrying optical power. The optical arrangement of such interferometers may be represented by Figure 1.

The interferometer is usually servo-locked on a dark fringe such that the output port is dark and the bright fringe travels towards the recycling mirror. Imperfect interference between the beams from the two arms of the interferometer causes light, that would have directed back towards the recycling mirror, to instead be split by the beamsplitter, half travelling towards the "dark fringe" output. This causes both loss in the circulating power build up and the increase of the photon noise level in the photo-detector of the “dark fringe" output.

One of the causes of imperfection in interference is birefringence of the beamsplitter which causes a change in the polarisation state of light of the beam of one arm ${ }^{5,6}$ leading, after recombination of the two beams, to a non-nil minimum-intensity at the "dark fringe" output. The deteriorated fringe contrast due to the birefringence in the beamsplitter may be written by the following formula given by Winkler ${ }^{7}$ :

$\frac{P_{\min }}{P_{\text {circ }}} \approx 10^{-3}\left(\frac{d \delta n}{\lambda / 100}\right)^{2}$

Where: $d$ is the thickness of the beamsplitter, 
$P_{\min }$ is the power transmitted to the "dark fringe" output, caused by the birefringence,

$P_{\text {circ }}$ is the circulating power in the interferometer,

$\delta n$ is the refractive index difference per unit length,

$d$ is the thickness of the beamsplitter.

The related phase shift (in degrees) between the two components is then:

$\varphi=360 \frac{d \delta n}{\lambda}$

Sapphire is an uni-axial crystal; a wave of any state of polarisation propagates along the c-axis without change. However, imperfect crystals, because of the non-constancy of the direction of the c-axis, show what we will call inhomogeneous birefringence in order to distinguish it from birefringence due to extrinsic effects such as mechanical stress or thermal stress.

In this paper, we present inhomogeneous and stress induced birefringence measurements on a high purity, polished $\mathrm{HEMEX}^{8}$ single crystal sapphire cylinder sample of $50 \mathrm{~mm}$ diameter and $100 \mathrm{~mm}$ length. Since the laser beam used has low power $(\sim 10 \mathrm{~mW})$ and the room temperature change is less than $2^{\circ} \mathrm{C}$ during the experiment, the birefringence due to thermal stress is negligible. An upper limit of the inhomogeneous birefringence is then deduced as well as its effect on the power loss in the interferometer. 


\section{Experimental Set up}

Figure 2 shows the experimental set up. A laser beam $(\lambda=1064 \mathrm{~nm})$ passes in succession through a polariser oriented at $45^{\circ}$ from the i-axis (horizontal), a photo-elastic modulator ${ }^{9}$ $\left(0^{\circ}\right.$ in respect to $\mathrm{i}$-axis $)$, a sapphire sample oriented in a manner to have its optical axis parallel to the beam and an analyser crossed with respect to the polariser $\left(-45^{\circ}\right)$.

The photo-elastic modulator $(\mathrm{PEM})^{10}$ converts the linearly polarised light from the polariser into elliptically polarised light oscillating between left and right ellipticity. The retardation in phase between the two components of the beam is taken to be

$$
\varphi_{P E M}=A_{0} \sin (\Omega t)
$$

where

$$
\begin{aligned}
& A_{0} \text { is the amplitude of the retardation. } \\
& \Omega=2 \pi f \mathrm{f} \text { is the frequency of the PEM oscillations, } 50 \mathrm{kHz} \text {. }
\end{aligned}
$$

This arrangement is very suitable for the measurement of a very low birefringence level, since the sensitivity of the PEM is less than $0.01^{\mathrm{o}} 11$

The amplitude of the wave after the polariser may be written

$$
E=E_{0}(\vec{i}+\vec{j}),
$$

which after passing through the PEM becomes: 


$$
E=E_{0}\left(\vec{i}+e^{i \varphi_{p e m}} \vec{j}\right)
$$

Note that because of the residual birefringence of the fused silica of the PEM, the equation (3) should be written as:

$$
\varphi_{P E M}=A_{0} \sin (\Omega t)+\varphi_{p e m 0} .
$$

Here $\varphi_{\text {pem } 0}$ is measured when there is no sapphire sample or by replacing the sample with a calibrated Babinet-Soleil Compensator. Previous authors ${ }^{10,}{ }^{11}$ have not included the residual birefringence due to the PEM.

The amplitude after passing through the sample becomes:

$$
E=E_{0}\left(\vec{i}+e^{i\left(\varphi_{p e m}+\varphi_{s}\right)} \vec{j}\right)
$$

where $\varphi_{s}$ is the retardation due to birefringence of the sapphire sample.

After passing through the analyser, the intensity on the photo-detector is then:

$$
I(t)=\frac{r I_{0}}{2}\left(1-\cos \left(\varphi_{s}+\varphi_{p e m 0}+A_{0} \sin (\Omega t)\right)\right.
$$

where $I_{0}$ is the intensity of the linearly polarized light from the polariser,

$\mathrm{r}$ is the factor loss due to surface reflection, absorption and scattering.

Equation (8) may be expressed as a Fourier series as follows:

$$
I(t)=\frac{r I_{0}}{2}\left(I_{D C}+I_{1 f} \cos (\Omega t)+I_{2 f} \cos (2 \Omega t)+\ldots\right) .
$$


where

$I_{D C}=1-J_{0}\left(A_{0}\right)$,

$I_{1 f}=2 \sin \left(\varphi_{S}+\varphi_{\text {pem } 0}\right) J_{1}\left(A_{0}\right)$,

$I_{2 f}=2 \cos \left(\varphi_{s}+\varphi_{p e m 0}\right) J_{2}\left(A_{0}\right)$.

The retardation of the sapphire sample can be deduced by taking the ratio of $\mathrm{I}_{1 \mathrm{f}}$ and $\mathrm{I}_{\mathrm{DC}}$ which leads to the following expression:

$\boldsymbol{\varphi}_{s}=\arcsin \left(\frac{I_{1 f}}{I_{D C}} \frac{1-J_{0}\left(A_{0}\right)}{2 J_{1}\left(A_{0}\right)}\right)-\varphi_{p e m 0}$,

The ratio is obtained by measuring the DC voltage with a voltmeter and the first harmonic of the voltage signal from a lock-in amplifier as shown in figure 2.

Since $\frac{I_{1 f}}{I_{D C}}=\frac{V_{1 f}}{V_{D C}}$, the retardation can be written as:

$\boldsymbol{\varphi}_{s}=\arcsin \left(\frac{V_{1 f}}{V_{D C}} \frac{1-J_{0}\left(A_{0}\right)}{2 J_{1}\left(A_{0}\right)}\right)-\varphi_{p e m 0}$

We can deduce from expression (10) that $\varphi_{s}$ is independent of light source fluctuations and the changes in the transmission function $r$, which leads to the high intrinsic accuracy of this method.

For practical reasons we set the amplitude $A_{0}$ of the modulated retardation of the PEM to $2.405 \mathrm{rd}\left(137.8^{\circ}\right)$, thus the DC signal strength is independent of the orientation of the sample with respect to the analyser. Equation (11) is then simplified to:

$\boldsymbol{\varphi}_{s}=\arcsin \left(\frac{V_{1 f}}{V_{D C}} \frac{1}{2 J_{1}\left(A_{0}\right)}\right)-\varphi_{p e m 0}$,

$\varphi_{\text {pem } 0}$ was measured to be $0.01^{\circ}$. 
The sapphire sample is held in a "V" shaped aluminium support (see Figure 3). The support and the sample are then set on a horizontal translation stage, with a step length of $\Delta x=0.5 \mathrm{~mm}$. The vertical scanning is done by adjusting the height of the $\mathrm{V}$ support with plates of different thickness, giving an average step length of about $5 \mathrm{~mm}$. Care was taken during the change of the vertical position to orient the sample to have its optical axis parallel to the beam. After every horizontal scan, the sample was rotated about $90^{\circ}$ in order to see if the birefringence pattern had changed. We found the same general pattern.

\section{Results and discussion:}

Figure 4 shows the phase shift due to the birefringence of the sapphire. We can notice the effect of the static mechanical stress in the hatched area in Figure 3, in which birefringence increases up to $2^{\circ}$. In the rest of the sample where the mechanical stress is lower, the birefringence remains less than $1^{\circ}$ as shown in Figure 5.

We can conclude from above that, as long as the beam passes through the central area, inhomogeneous and stress induced birefringence is less than $1^{\circ}$ for a sample thickness of $10 \mathrm{~cm}$. This leads, using the equation (1), to degradation in the fringe contrast of $7.10^{-5}$.

The loss caused by birefringence should be compared with other sources of loss in the interferometer, which are different for the two designs of interferometer currently under construction: Fabry-Perot Michelson (LIGO and VIRGO) and few-bounce, high

recycling factor systems (GEO). In the former, birefringence losses present in 
beamsplitter and input mirrors should be compared to mirror coating and the other optical losses. Table 1 summarizes the expected losses during one round trip from various sources for fused silica and sapphire substrates in an interferometer illuminated by a light power of 100W and with 100 bounces in each Fabry-Perot cavity. The thickness of each transmitting component (beamsplitter and input mirrors) is taken to be $10 \mathrm{~cm}$. The thermal lensing is estimated using the approximation given by Winkler ${ }^{7}$ of the full analytical calculation of Hello and Vinet ${ }^{12}$. This gives the change of the optical path by $\delta s \approx 1 . .3 \frac{\beta}{4 \pi \kappa} p_{a} d$ with a relative error less than $30 \%$ (of the same order as the estimates of the other quantities in the table). Here $\beta$ is the temperature dependence of the index of refraction, $\kappa$ is the thermal conductivity, $p_{a}$ is the power absorbed per unit length and $d$ is substrate thickness. Then the fractional loss can be deduced from the equation (1) by substituting $d \delta n$ with $\delta s$.

Few bounce designs are likely to be limited by wave-front curvature mismatch caused by mirror curvature mismatch and astigmatism and possibly alignment fluctuations. The fractional power loss ${ }^{13}$, due to angular misalignment $\vartheta$ between the interfering beams at the beamsplitter is $\sim\left(\frac{\boldsymbol{\theta}}{\boldsymbol{\theta}_{c}}\right)^{2}$, where, and $\vartheta_{c}=\frac{\lambda \sqrt{2}}{\pi \omega}$ is the beam divergence. Thus, a power loss of the same order of the beamsplitter birefringence would be caused, in an interferometer of $1 \mathrm{~km}$ arm length and $1064 \mathrm{~nm}$ of laser wavelength, by a misalignment of $10^{-7} \mathrm{rad}$

The losses can be estimated for future experiments: the highest recycling factor demonstrated thus far ${ }^{14}$ is 300 from a contrast of 0.994 - the loss coming from mirror 
astigmatism. This could conceivably be reduced by a factor of 100 by alignment of the astigmatic axes (if symmetrical) which would place birefringence losses approximately 3 times higher than these. Alignment fluctuations are servo- and suspension system dependent. Automatic alignment systems have achieved this in a cavity suspended from single pendulums ${ }^{15}$. To achieve the necessary orientation control in a multistage isolation system $^{16}$, will require a reduction of the low frequency orientation noise by a factor of 100 - by using either higher servo gain or a preisolation system.

We conclude that in an interferometer with cavities in the arms, substrate birefringence is similar in magnitude to other sources of loss. In a few-bounce system, misalignment and wave-front curvature mismatch losses are likely to be similar in magnitude to birefringence-induced losses.

In either case birefringence losses are not likely to be the dominant loss mechanism though a reduction through stress-birefringence minimisation and high crystal quality would be beneficial.

Moreover, in order to compare the stress-induced birefringence in sapphire and fused silica, we have made an estimation of the ratio of mechanical stress-induced birefringence in sapphire to that induced in fused silica using the photo-elastic effect theory ${ }^{17}$ in a simple case where a tensile stress, $\sigma_{z}$ is applied in the direction of the optical axis of the crystal. The mechanical stress-induced birefringence for both fused silica and sapphire, may be written as: 
$\Delta n_{f-\text { silica }}=\frac{1}{2} n^{3} \frac{c_{11}+2 c_{12}}{\left(c_{11}-c_{12}\right)\left(c_{11}+c_{12}\right)}\left(p_{12}-p_{11}\right) \sigma_{z}$,

$\Delta n_{\text {sapphire }}=\frac{1}{2} \frac{\left[n_{o}{ }^{3}\left(\left(c_{11}+c_{12}\right) p_{12}-2 c_{13} p_{31}\right)-n_{e}{ }^{3}\left(\left(c_{11}+c_{12}\right) p_{13}-c_{13}\left(p_{11}+p_{12}\right)\right)\right]}{-2 c_{13}{ }^{2}+c_{11} c_{33}+c_{12} c_{33}} \sigma_{z}$,

Where:

$p_{i j}$ are the tensor components for the phtoelastic effect,

$c_{i j}$ are the elastic stiffness coefficients,

$\sigma_{z}$ is a tensile stress applied on $\mathrm{z}$ direction, taken parallel to c-axis

The calculation of the ratio $\frac{\Delta n_{f-s i l i c a}}{\Delta n_{\text {sapphire }}}$ leads to a mechanical stress induced birefringence in the fused silica 4.3 times larger than in sapphire for the same mechanical conditions.

Similarly, using the formula given by Winkler ${ }^{9}$ we found that the ratio of thermal stress induced birefringence in fused silica and the one in sapphire can be approximated by:

$\frac{\Delta n_{\text {sapphire }}}{\Delta n_{f-\text { silica }}} \approx \frac{\kappa_{\text {sapphire }}}{\kappa_{f-\text { silica }}} \frac{\alpha_{f-\text { silica }}}{\boldsymbol{\alpha}_{\text {sapphire }}}$,

Where:

$\kappa$ is the thermal conductivity,

$\alpha$ is the thermal expension.

This gives a thermal stress-induced birefringence in fused silica 2.4 times higher than in sapphire.

\section{Conclusion:}

The results obtained on the sapphire birefringence are very promising. Indeed, the level of inhomogeneous birefringence is comparable to that in fused silica ${ }^{18}$. Moreover, with the excellent mechanical and thermal properties, we can expect the stress-induced birefringence in a sapphire beamsplitter, and also in the input mirrors in a Fabry-Perot configuration, to be lower than in one made of fused silica. However, further 
investigation on both thermal and mechanical stress-induced birefringence on sapphire must be done with the finite element method to have definitive results.

A computer controlled automatic birefringence measurement facility is under development. This will allow thorough and more accurate mapping of birefringence of different sapphire samples under different stress conditions.

\section{Acknowledgement:}

We would like to thank C.Boccara (ESPCI, Paris) for useful suggestions on the optical setup, and P. Hello (GROG, Orsay) for his fruitful comments on this paper. We thank John Winterflood and C.N Zhao for technical assistance and Mike Tobar for the loan of his sapphire crystal. We also thank the workshop staff . This work was supported by the Australian Research Council 


\section{Figure captions:}

\section{Figure 1:Optical layout of an interferometer using power recycling:}

RM: Recycling mirror, BS: Beamsplitter M1: mirror of arm 1, M2: mirror of arm 2.

DF Output : “dark fringe” output.

\section{Figure 2: The experimental arrangement:}

a) Nd:YAG laser $(1064 \mathrm{~nm})$ with incident power of $10 \mathrm{~mW}$.

b) The polariser and analyser are Glan-Thomson polarisers, set in crossed position with respect to one another.

c) The PEM is excited by a voltage signal of $50 \mathrm{kHz}$ which is also the external source of the lock-in amplifier.

d) The signal coming from the photodetector is AC signal with a DC level (Vdc) detected by a voltmeter. The lock-in amplifier gives the RMS value of the first harmonic (V1f).

\section{Figure 3: the sapphire sample on its V support:}

The laser beam is normally incident on one of the face of the sample and parallel to its optical axis. 


\section{Figure 4: The birefringence of the HEMEX sapphire sample:}

Plot of the phase shift due to the birefringence of the $100 \mathrm{~mm}$ length sapphire sample. The measurement steps are $0.5 \mathrm{~mm}$ and $5 \mathrm{~mm}$ for the horizontal axis and the vertical axis consecutively. The area with high phase shift (about $2^{\circ}$ ) corresponds to the hatched area shown in Figure 3.

Figure 5: Birefringence of the HEMEX sample in the central area.

Plot of the phase shift du to the birefringence in the central area of sapphire. 


\section{Table Captions:}

Table1: Fractional power loss during one round trip in the transmitting components in Fabry-Perot configuration.

The values reported in this table are deduced or measured as follows: The number in bracket is the fractional loss each time a beam encounters a surface/substrate. The multiplier is the number of times a beam experiences the loss. The factor $\frac{1}{2}$ is due to the fact that the incident power being split into two arms.

a) The birefringence in silica is taken from Logan's measurement ${ }^{6}$.

b) The evaluation of the substrate loss is done assuming an absorption coefficient of 3.ppm/cm for sapphire measured by Blair et al ${ }^{3}$, and $1 \mathrm{ppm} / \mathrm{cm}$ for a fused silica measured by VIRGO ${ }^{19}$

c) The thermal lensing is estimated using the method mentioned in section 3 .

d) The scattering loss is deduced from the measurements reported by Crystal Systems ${ }^{20}$ for the sapphire $(20 \mathrm{ppm} / \mathrm{cm})$ and by Baures for the fused silica $(2 \mathrm{ppm} / \mathrm{cm})^{21}$

e) We assume a mirror coating for both sapphire and fused silica test masses with $10^{-6}$ fractional power loss per bounce. 


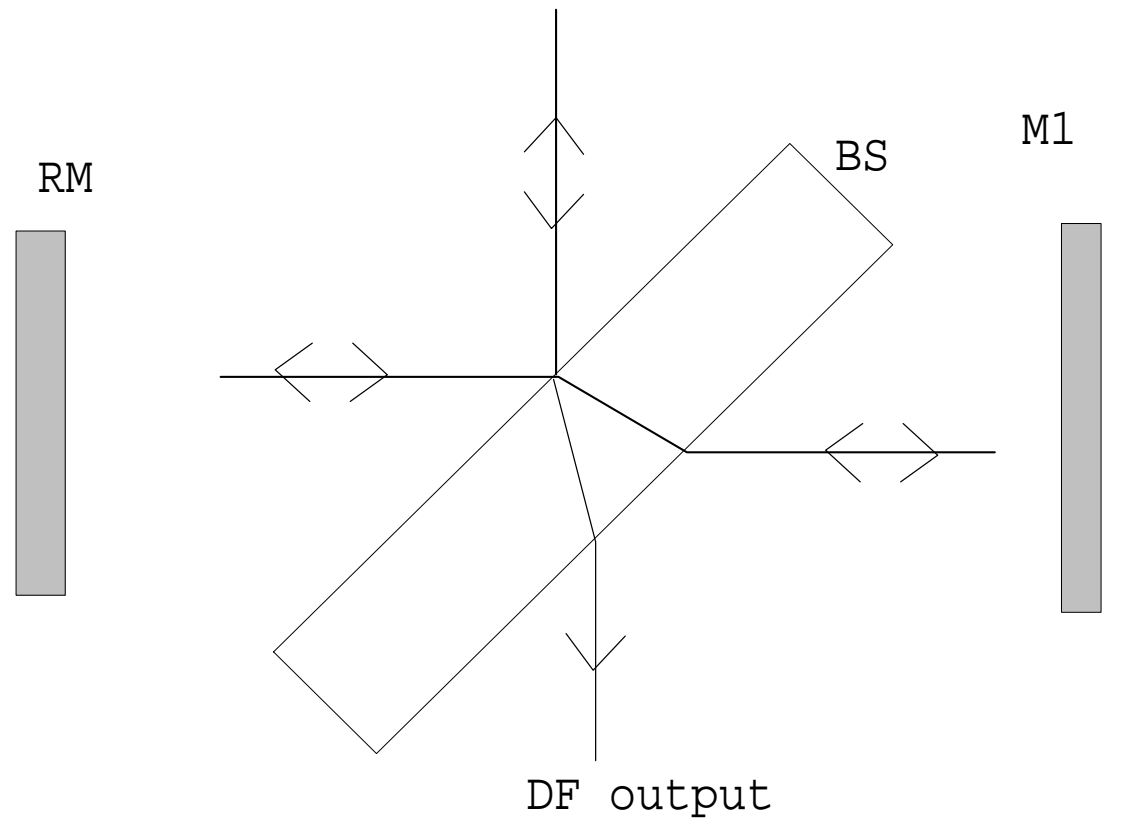

Figure 1 


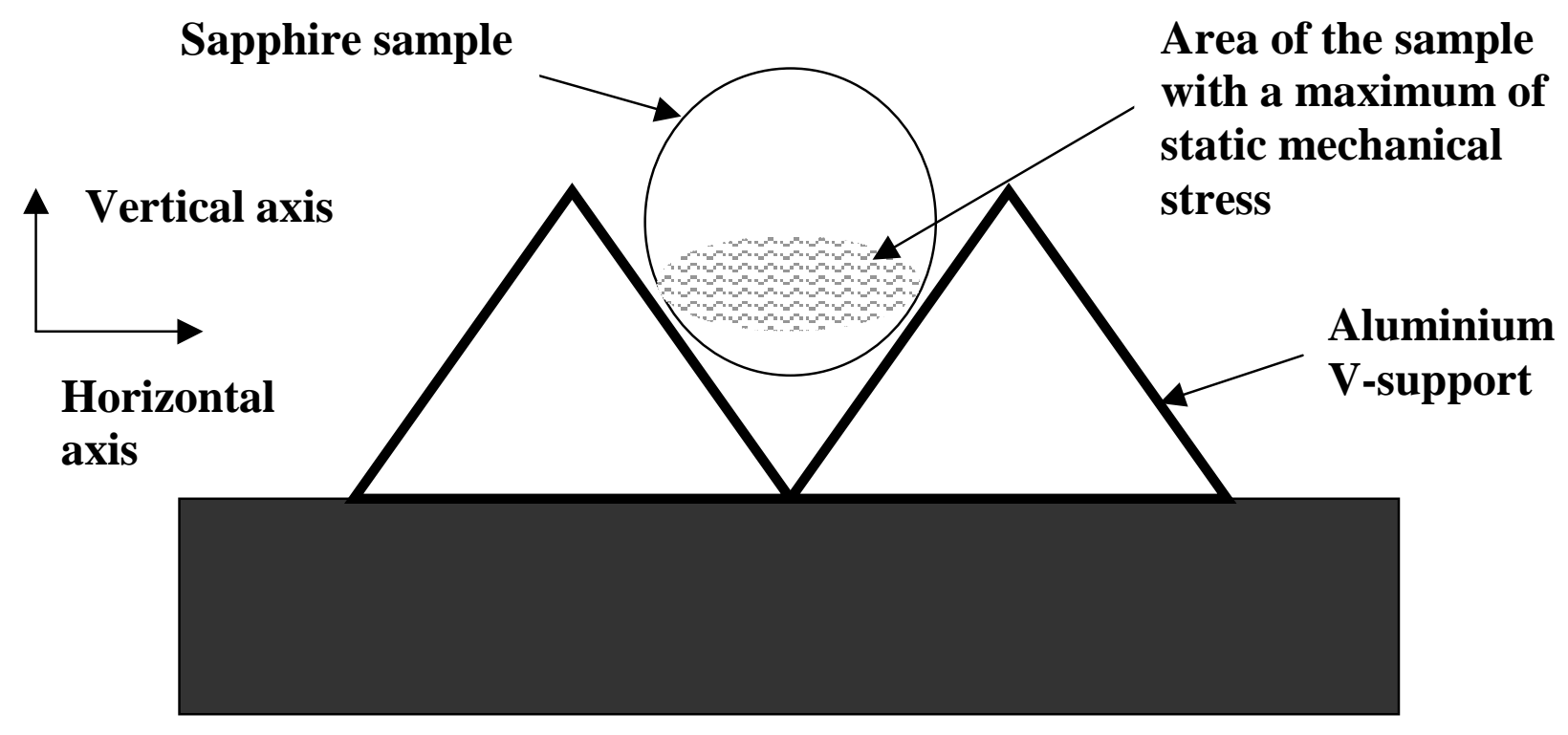

Figure 3 


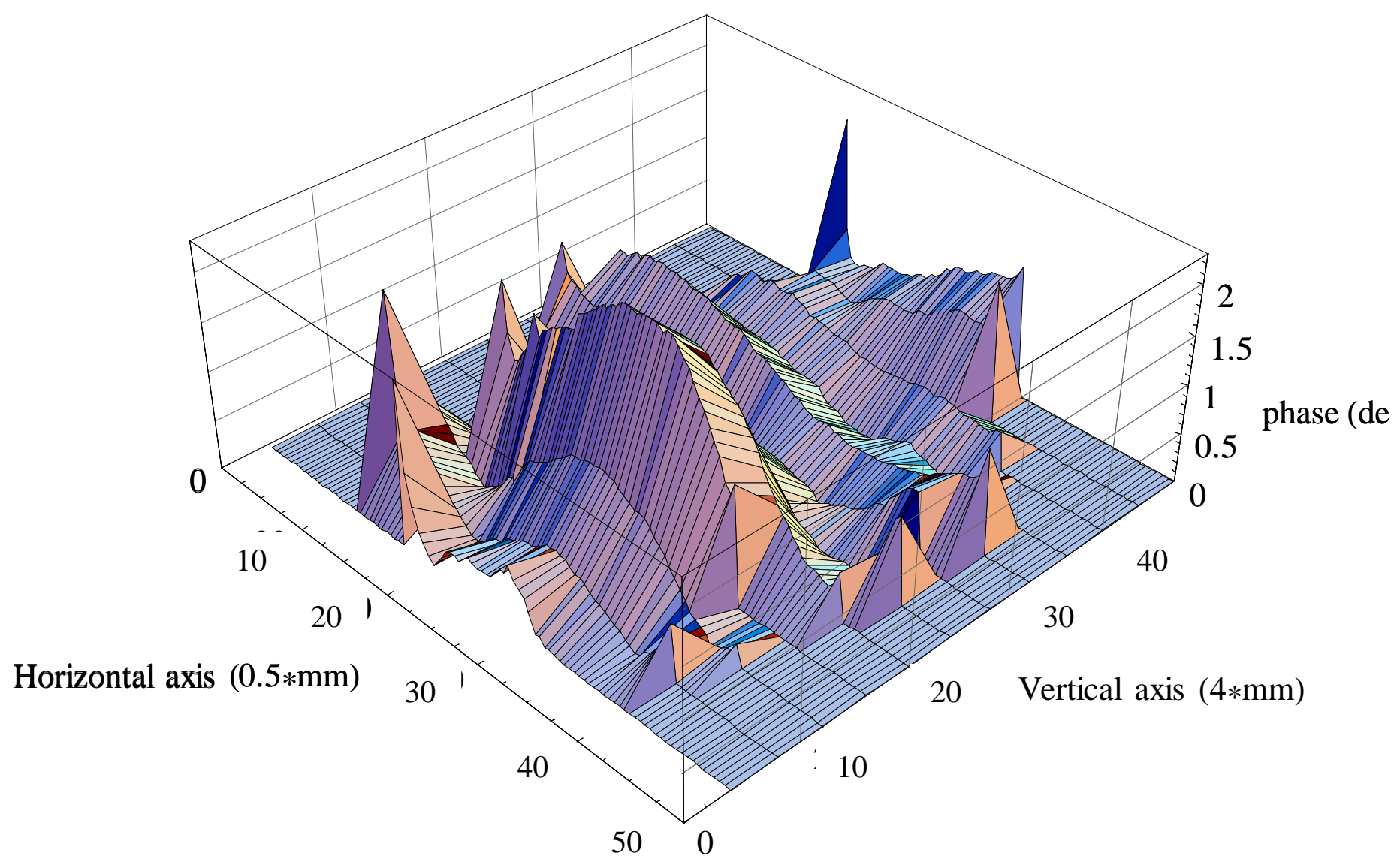

Figure 4 


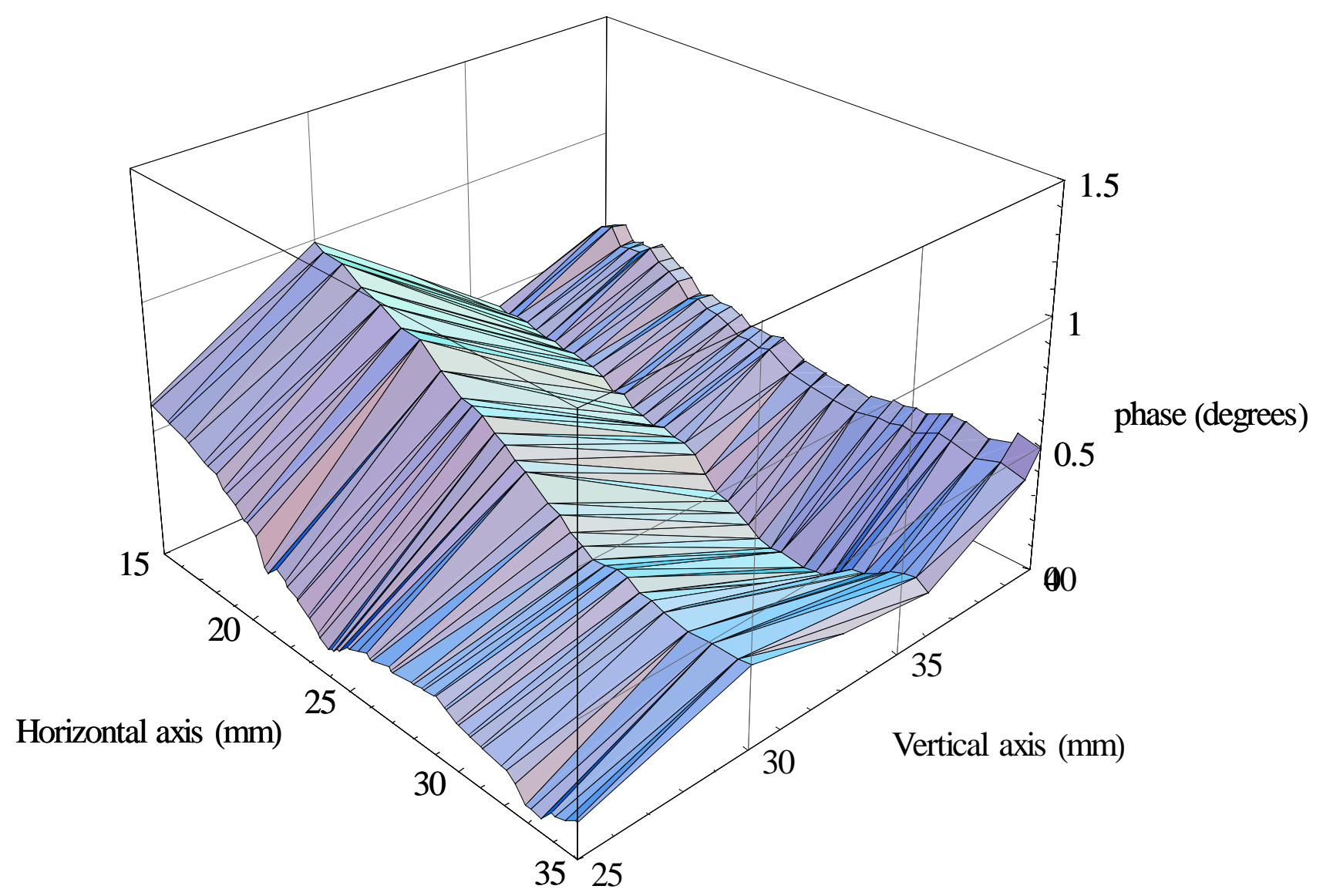

Figure 5 


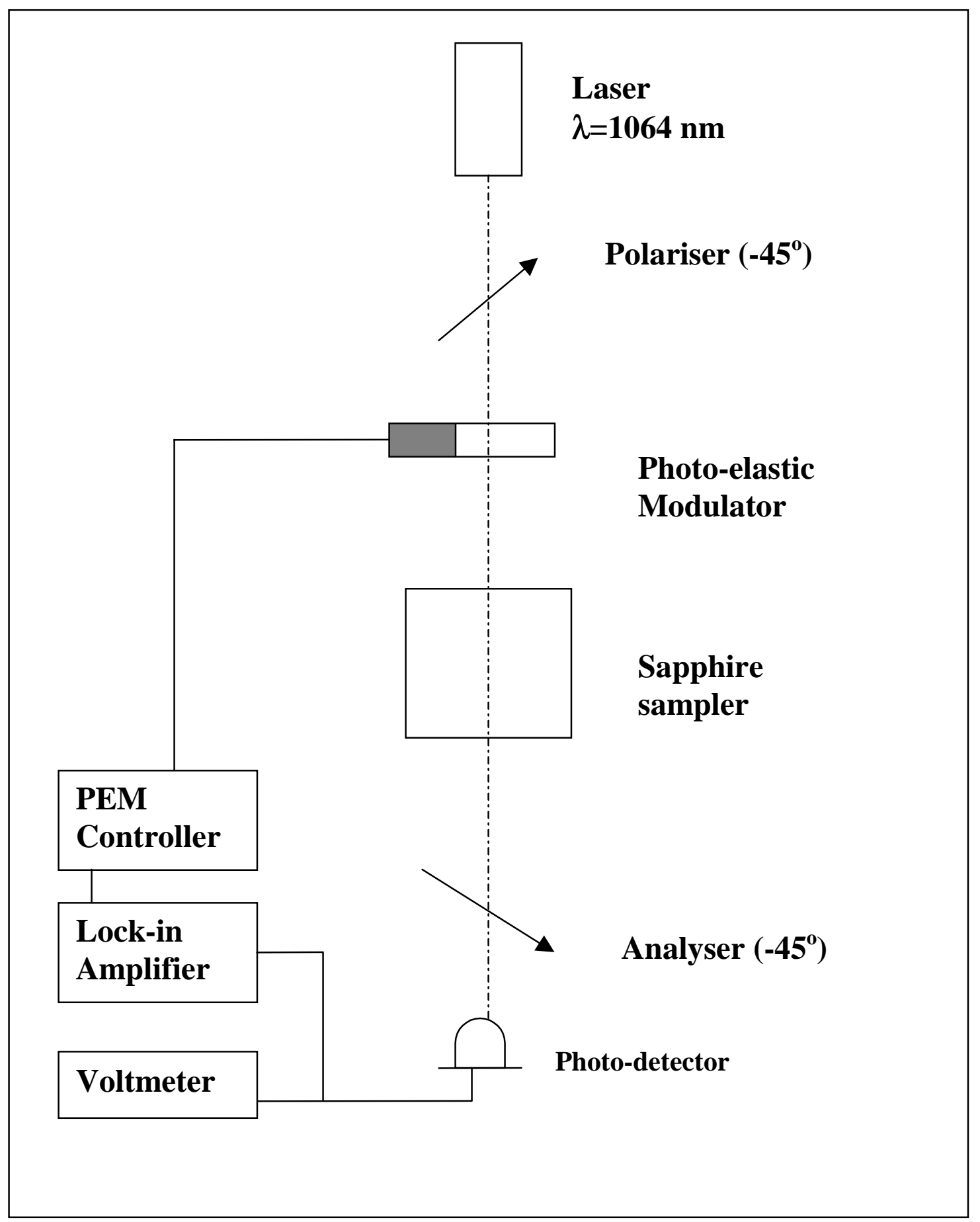

Figure 2 


\begin{tabular}{|l|c|c|}
\hline & Sapphire & Fused Silica \\
\hline a) Substrate birefringence & $\frac{6}{2} \times\left(7.10^{-5}\right)=2.1 \times 10^{-4}$ & $\frac{6}{2} \times\left(5.10^{-4}\right)=15 \times 10^{-4}$ \\
\hline b) Substrate absorption & $\frac{6}{2} \times\left(3.10^{-5}\right)=9 \times 10^{-5}$ & $\frac{6}{2} \times\left(10^{-5}\right)=3 \times 10^{-5}$ \\
\hline c) Substrate thermal lensing & $\frac{6}{2^{2}} \times\left(4.10^{-7}\right)=6 \times 10^{-7}$ & $\frac{6}{2^{2}} \times\left(10^{-5}\right)=1.5 \times 10^{-5}$ \\
\hline d) Substrate scattering & $\frac{6}{2} \times\left(210^{-4}\right)=6 \times 10^{-4}$ & $\frac{6}{2} \times\left(210^{-5}\right)=6 \times 10^{-5}$ \\
\hline e) Coating absorption & $\frac{4 \times 100}{2} \times\left(10^{-6}\right)=2 \times 10^{-4}$ \\
\hline
\end{tabular}

\section{Table 1}




\section{References}

${ }^{1}$ M.Taniwaki et al., High quality factor measurements of sapphire for the test masses of a laser interferometer gravitational wave detector, submitted to Phys. Lett. A, April 1997.

${ }^{2}$ V.B.Braginskii et al, Systems with Small Dissipation (The University of Chicago Press, Chicago, 1985).

${ }^{3}$ D.G.Blair, "Optical Absorption Measurements in Monocrystalline Sapphire at 1 micron", Journal of Optical Materials (1997) to be published.

${ }^{4}$ B.J.Meers, Phys. Rev. D 38 (1988) 2317.

${ }^{5}$ L.Ju et al., Phys. Lett. A 218 (1996) 197.

${ }^{6}$ J.Logan et al , Optics Communications 107 (1994) 342.

${ }^{7}$ W.Winkler et al, Phys. Rev. A 44 (1991)7022.

${ }^{8}$ Crystal Systems Inc, 27 Congress St, MA 01970 U.S.A.

${ }^{9}$ Hinds Instruments Inc,. Hillsboro OR

${ }^{10}$ J.C.Canit and J.Badoz, Appl. Opt. 22 (1983) 582

${ }^{11}$ T.C Oakberg, Measurement of low-level strain birefringence in optical elements using a phtoelastic modulator, Hillsboro OR: Hinds instruments Inc.

${ }^{12}$ P. Hello and J.Y. Vinet, J. Phys. (Paris) 51, 2243 (1990).

${ }^{13}$ B.J.Meers and K.A.Strain, Phys. Rev. D 43 (1991) 3117.

14 D.Schnier et al, Phys. Lett. A 225(1997) 210.

${ }^{15}$ Euan Morrison et Al, Appl. Opt 33 (1994) 5037.

${ }^{16}$ L. Ju and D. Blair, Rev. Sci. Instrum. 65 (11), November 1994.

${ }^{17}$ S.Huard, Polarisation of Light (John Wiley\&Sons Ltd, Chichster, 1997) , p157.

${ }^{18}$ W.Winkler et al, Optics communications 112 (1994) 245.

19 VIRGO project, privat communications (1996). 
${ }^{20}$ Crystal Systems private communications (1995), which referenced W.J. Tropf and M.E Thomas at The Johns Hopkins University.

${ }^{21}$ P.Y. Baures and C.N. Man, Opt. Mater. 2(1993) 241. 